\title{
Desenvolvimento de mousse de maracujá sem ingredientes de origem animal
}

Fernanda Germano Alves Gautério, Mariane Igansi Alves, Matheus Ludtke Lauffer, Karine Laste Macagnan, Claire Tondo Vendruscolo, Angelita da Silveira Moreira

https://doi.org/10.4322/mp.978-65-991393-5-2.c2

\section{Resumo}

O objetivo do trabalho foi o desenvolvimento e a caracterização de mousse de maracujá sem ingredientes de origem animal, sob a influência da associação de dois tipos de hidrocoloides: xantana (xantana pruni - produzida por Xanthomonas arboricola pv pruni ou xantana comercial), e guar. Buscando atender ao nicho de mercado dos consumidores que, por questões de saúde ou ideologia, não ingerem produtos de origem animal, duas formulações básicas de mousse de maracujá - uma tradicional, para fins comparativos, e uma alternativa, com variação da procedência da xantana, foram estudadas. Primeiramente, realizou-se 3 ensaios sensoriais, nos quais avaliouse: variação das concentrações de xantana e guar e, após, variação das concentrações de açúcar e suco de maracujá na formulação alternativa; e comparação entre as mousses sem ingredientes de origem animal formuladas com xantana pruni:guar e xantana comercial:guar, e a tradicional. Na sequência, as mousses foram avaliadas quanto às medidas reométricas (viscosidade e viscoelasticidade) e cor instrumental. Observou-se que a mousse alternativa formulada com xantana pruni apresentou potencial de comercialização, pois teve índice de aceitabilidade e intenção de compra semelhantes à mousse tradicional; porém, superiores à mousse com xantana comercial.

Palavras-chave: sobremesa de maracujá, intolerantes à lactose, xantana, guar, Xanthomonas arboricola pv pruni.

\section{Introdução}

Mousse consiste em uma sobremesa láctea, obtida a partir de uma emulsão do tipo óleo em água (O/A), estabilizada por proteínas, e incorporada de ar pela formação de espuma mediante batimento mecânico da massa (OHATA et al., 2005). Segundo McClements (2005), a emulsão pode ser definida como uma mistura entre dois líquidos imiscíveis em que um deles, chamado de fase dispersa, encontra-se na forma de finos glóbulos no meio do outro líquido, esse denominado de fase contínua.

O Brasil é o maior produtor mundial de maracujá, atingindo em 2019 uma produção de 593.429 toneladas (IBGE, 2019), sendo essa fruta comercializada principalmente na forma de suco. A partir do suco podem ser elaborados diversos produtos como, por exemplo, refrescos em pó, néctares, sorvetes, geleias, entre outros; sendo a mousse de maracujá uma alternativa para agregar valor e aumentar o consumo dessa fruta. 
A legislação brasileira não define um padrão de identidade para a mousse de maracujá, em virtude de ser considerada uma sobremesa de consumo caseiro; porém, também é servida em estabelecimentos comerciais (FOLEGATTI, 2001). Apesar de se verificar uma diversidade em termos de ingredientes nas formulações tradicionais das receitas de mousse, por ser uma sobremesa essencialmente láctea; não pode ser ingerida por consumidores que apresentem restrições alimentares por fatores fisiológicos, como alergia ou intolerância alimentar, ou por questão cultural ou ideológica quanto à ingestão de produtos de origem animal.

O consumo de determinados alimentos pode causar intolerância ou alergia, como é o caso do leite e de seus derivados, pela presença da lactose e caseína. A intolerância a esse carboidrato, por exemplo, é causada pela deficiência da enzima lactase; sem esta, a lactose não pode ser digerida no organismo, causando sintomas gastrintestinais após a ingestão, como dores abdominais e diarreia (DI COSTANZO; CANANI, 2018). O Veganismo é uma filosofia que envolve dieta e estilo de vida; é movido por convicções éticas com base nos direitos dos seres vivos. Adeptos dessa filosofia não consomem quaisquer produtos de origem animal, inclusive gelatina animal (LONDERO, 2019). Ainda, para algumas pessoas, também existe uma preocupação em reduzir o teor calórico e/ou teor de gorduras saturadas e colesterol dos alimentos através da substituição ou eliminação dessas gorduras em busca de uma alimentação mais saudável.

Para atender às exigências dos consumidores portadores de restrições alimentares por fatores fisiológicos, ou ainda adeptos do Veganismo, bem como às deficiências do mercado, as indústrias alimentícias vêm desenvolvendo produtos e tecnologias para elaboração de alimentos desprovidos de ingredientes potencialmente alergênicos ou de origem animal de modo geral. Dessa forma, é imprescindível a substituição de alguns ingredientes tradicionais por outros de características tecnológicas semelhantes, obtendo-se, assim, um produto diferenciado, mas com equivalente qualidade.

De acordo com Dickinson (2003), hidrocoloides polissacarídicos são considerados ingredientes de alta tecnologia nas indústrias alimentícias, podendo atuarem como espessantes e estabilizantes e, inclusive, virem a substituir um dos ingredientes mais importantes da mousse, a gelatina. São amplamente utilizados como aditivos na tecnologia de alimentos com a finalidade de estabilizar emulsões O/A e controlar suas propriedades reológicas (ROJAS; ROSELL; BARBER, 1998; PARASKEVOPOULOU et al., 2003); estando a goma guar e a goma xantana entre os hidrocoloides polissacarídicos mais utilizados (DEMIRCI; YILMAZ; DEMIRCI, 2014).

A xantana é um importante aditivo industrial de origem microbiana (LILLY; WILSON; $\mathrm{LEACH}, 1958)$, de alta viscosidade, mesmo quando em baixas concentrações, e comportamento pseudoplástico, que reduz a viscosidade com aumento da taxa de deformação. Possui propriedades emulsificantes, suspensivas e estabilizantes, além de manter essas características em uma ampla faixa de $\mathrm{pH}$ e temperatura (LUVIELMO; SCAMPARINI, 2009); características essas muito valorizadas nas indústrias alimentícias. Por tais qualidades, e por ser compatível com os ingredientes alimentícios, apresenta bons resultados quando utilizada na elaboração de mousse, auxiliando na aeração, estabilização, textura e viscosidade do produto (LUCCA; TEPPER, 1994). Uma importante característica dessa goma é a sinergia que ela possui com galactomananas, como a goma guar. Quando associadas, essas gomas agem sinergicamente, aumentando de modo significativo a viscosidade dos alimentos aos quais são adicionadas (GARCÍA-OCHOA et al., 2000). 
A goma guar é um aditivo polissacarídico extraído do endosperma vegetal da espécie Cyamoposis tetragonolobus. Esse hidrocoloide, quando em contato com a água, forma um gel altamente viscoso. Entretanto, muitas vezes, essa elevada viscosidade tem dificultado a aplicação dessa goma; sendo necessário proceder a hidrólise parcial, onde parte de suas moléculas são quebradas, transformando-a em um oligossacarídeo, de forma a gerar soluções menos viscosas (ROJAS; ROSELL; BARBER, 1998). Outra forma de diminuir esse inconveniente é a sua utilização combinada com outros polímeros em formulações alimentícias (SILVA et al., 2018).

A preocupação com a saúde vem alterando os hábitos alimentares dos consumidores, que buscam cada vez mais produtos diferenciados. Porém, ainda se observa a carência de produtos destinados aos consumidores com dietas restritivas. Ingredientes como o extrato hidrossolúvel de soja e polissacarídeos, como a goma guar e outras, têm algumas características tecnológicas similares às das proteínas lácteas e gordura, respectivamente; são utilizadas na elaboração de produtos industriais como o creme de soja, um substitutivo do creme de leite tradicional. Esse, por sua vez, pode ser empregado na elaboração de sobremesas do tipo mousse.

Neste contexto, este trabalho objetivou o desenvolvimento e a caracterização de mousse de maracujá sem ingredientes de origem animal, sob a influência da associação de dois tipos de hidrocoloides:xantana (xantana pruni - produzida por Xanthomonas arboricola pv pruni ou xantana comercial) e guar, em comparação com a formulação tradicional.

\section{Materiais e Métodos}

\subsection{Elaboração das mousses de maracujá}

A base das formulações tradicional e sem ingredientes de origem animal das mousses de maracujá foi desenvolvida anteriormente por Lauffer et al. (2010). Os ingredientes utilizados na formulação tradicional (Tabela 1) foram: leite condensado (Batavo ${ }^{\circledR}$ ), creme de leite $\left(\right.$ Batavo $^{\circledR}$ ), suco de maracujá reconstituído (Maguary ${ }^{\circledR}$ ), água e gelatina em pó sem sabor e incolor (Madrugada ${ }^{\circledR}$ ). As mousses alternativas (Tabela 1 ) foram elaboradas empregando açúcar de cana refinado $\left(\right.$ Caravela $\left.^{\circledR}\right)$, creme de soja $\left(\right.$ Batavo $\left.^{\circledR}\right)$, suco de maracujá reconstituído $\left(\right.$ Maguary ${ }^{\circledR}$ ), água e uma associação de 2 hidrocoloides, xantana e guar $\left(\right.$ Germinal $\left.^{\circledR}\right)$.

Nessa formulação foi variada a procedência da xantana: (A) produzida no Laboratório de Biopolímeros/CDTec/UFPel, por Xanthomonas arboricola pv pruni, cepa EDE, que a seguir será denominada apenas por xantana pruni, e (B) comercial (Farmaquímica ${ }^{\circledR}$ ). As mousses foram elaboradas a partir da pesagem, e batimento mecânico de todos os ingredientes em equipamento do tipo mixer (Mallory modelo Roboty 250), na velocidade máxima por 5 minutos.

\subsection{Análise sensorial das mousses de maracujá}

Para as análises sensoriais, amostras de $15-20 \mathrm{~g}$ das mousses foram acondicionadas em copos brancos de polietileno, codificadas aleatoriamente com três dígitos e armazenadas por $24 \mathrm{~h}$ a $4{ }^{\circ} \mathrm{C}$. As análises foram realizadas em dias alternados, no turno da manhã (9-11 h) em cabines isoladas, sob luz branca. As amostras foram ofertadas à temperatura ambiente a 55 julgadores não treinados da comunidade da 
Universidade Federal de Pelotas - UFPel, os quais apresentavam idade entre 18 e 47 anos.

Tabela 1. Composição das formulações alternativa básica* e tradicional das mousses de maracujá.

\begin{tabular}{cc}
\hline \multicolumn{1}{c}{ Ingredientes } & Concentração (\%) \\
\hline \multicolumn{1}{c}{ Formulação alternativa } & \\
\hline Açúcar & 27 \\
Suco de maracujá & 25 \\
Creme de soja & 40 \\
Agente estabilizante (xantana:guar) & $x^{\star *}$ \\
Água & qsp \\
\hline Formulação tradicional \\
\hline Leite condensado \\
Creme de leite \\
Suco de maracujá \\
Gelatina \\
Água \\
\hline
\end{tabular}

*de acordo com Lauffer et al. (2010)

** variado de acordo com o ensaio sensorial 1

*** quantum satis per

As determinações sensoriais foram estruturadas em 3 ensaios: ensaio 1) variação da concentração da associação das gomas xantana pruni e guar na formulação alternativa de mousse (Tabela 2); ensaio 2) variação das concentrações de açúcar e suco de maracujá na formulação alternativa preparada com a proporção de gomas selecionadas no ensaio 1 (Tabela 3); ensaio 3) variação da procedência da xantana (xantana pruni e comercial) e comparação com a formulação tradicional de mousse de maracujá.

\subsubsection{Testes sensoriais}

Nos 3 ensaios sensoriais foi aplicado o Teste de Ordenação, sendo solicitado aos julgadores que ordenassem de forma decrescente a preferência das amostras; atribuindo nota 1 à amostra mais preferida e 5 (ensaio 1) ou 4 (ensaio 2) ou 3 (ensaio 3) a menos preferida. Os resultados desse teste foram tratados pelo Teste de Friedman e, posteriormente, foi determinada a diferença mínima significativa (dms) entre as amostras, a um nível de significância de $5 \%$. 
O Teste de Aceitação também foi aplicado nos 3 ensaios sensoriais, em que os julgadores receberam uma ficha de avaliação contendo a escala hedônica estruturada de 9 pontos, ancorados em seus extremos nos termos desgostei muitíssimo (1) a gostei muitíssimo (9) para avaliação dos atributos textura e sabor das mousses de maracujá. Com esses resultados foram calculados os Índices de Aceitabilidade (IA), onde a nota máxima (9) correspondeu a 100\% de aceitabilidade, e a média das notas dadas pelos julgadores para cada atributo definiu o IA.

No ensaio sensorial 3 foi aplicado o Teste de Intenção de Compra, além dos Testes de Ordenação e de Aceitação. No Teste de Intenção de Compra os julgadores receberam uma ficha contendo uma escala estruturada de 5 pontos, ancorados em seus extremos nos termos certamente não compraria (1) a certamente compraria (5); com os resultados, foi calculada a porcentagem de Intenção de Compra para cada amostra através da porcentagem dos escores, considerando o 5 (certamente compararia) como $100 \%$ e a média das notas dadas pelos julgadores definindo a porcentagem de intenção de compra.

No ensaio sensorial 1 foram apresentadas aos julgadores 5 amostras de mousse de maracujá alternativa, com 5 diferentes associações das gomas xantana pruni e guar (Tabela 2); a fim de avaliar qual seria a melhor proporção das mesmas. Com a proporção de hidrocoloides (xatana pruni:guar) selecionada, realizou-se o ensaio sensorial 2, em que estudou-se 4 combinações de concentrações de açúcar (27 e $33 \%$ ) e de suco de maracujá (22 e $25 \%$ ) na formulação alternativa de mousse (Tabela 3). Partindo da melhor proporção de hidrocoloides xantana pruni:guar e, também, com as melhores concentrações de açúcar e de suco de maracujá na formulação alternativa de mousse, realizou-se o ensaio sensorial 3.

Nesse ensaio, 3 formulações de mousse foram analisadas: 1 tradicional e 2 alternativas (em que variou-se a procedência da goma utilizada: (A) xantana pruni (VENDRUSCOLO; MOREIRA; VENDRUSCOLO, 2006) e (B) xantana comercial), com a finalidade de comparar a preferência e a aceitabilidade das formulações alternativas e tradicional das mousses de maracujá.

\subsection{Determinações físicas}

\subsubsection{Medidas reométricas}

As medidas reométricas das mousses de maracujá do ensaio sensorial 1 compreenderam as curvas de viscosidade e de viscoelasticidade, sendo essas determinadas em reômetro (HAAKE RheoStress, modelo RS150) a $25^{\circ} \mathrm{C}$, utilizando dispositivo de medida placa-placa PP35Ti. As medidas de viscosidade foram realizadas no modo rotativo, com taxa de deformação de 0,1 a $100 \mathrm{~s}^{-1}$. A viscoelasticidade foi determinada através de medidas oscilatórias, com frequência variando de 1 a $15 \mathrm{~Hz}$ em taxa de deformação de $50 \mathrm{~Pa}$.

\subsubsection{Determinação instrumental da cor}

A determinação instrumental da cor das amostras de mousse de maracujá referentes ao ensaio sensorial 3 foi realizada em colorímetro (Minolta ${ }^{\circledR}$, modelo CR-300). Os parâmetros de cor medidos foram: a luminosidade $L^{*}(0=$ preto e $100=$ branco $)$ e as coordenadas de cromaticidade $a^{*}\left(+a^{*}=\right.$ vermelho $\mathrm{e}-\mathrm{a}^{*}=$ verde $)$ e $\mathrm{b}^{*}\left(+\mathrm{b}^{*}=\right.$ amarelo $\mathrm{e}$ $\left.-b^{\star}=a z u l\right)$. Os parâmetros de cor foram convertidos em ângulo de cor Hue, que indica 
a tonalidade da cor das amostras $\left(0^{\circ}\right.$ ou $360^{\circ}=$ vermelho; $90^{\circ}=$ amarelo; $180^{\circ}=$ verde; $270^{\circ}=$ azul), sendo calculado por $\mathrm{H}^{\circ}=\tan ^{-1}(\mathrm{~b} / \mathrm{a})$. Também foi calculado o parâmetro Croma $\left(C^{\star}\right)$, que indica a intensidade da cor das amostras, por $C^{*}=\left(a^{\star^{2}}+b^{\star^{2}}\right)^{1 / 2}$ (ZHAN et al., 2008).

\section{Resultados e Discussão}

A Tabela 2 mostra o somatório das notas atribuídas às amostras de mousse de maracujá com variação na concentração das gomas xantana pruni e guar (ensaio 1) no Teste de Ordenação de preferência para cada amostra estudada.

$\mathrm{Na}$ ordenação, o Teste de Friedman não revelou diferença significativa de preferência, a um nível de significância de $5 \%$, entre as 5 amostras avaliadas $\left(x_{0}<x^{2}\right)$; demonstrando que as associações foram igualmente preferidas pelos julgadores. Pelo somatório da ordenação (Tabela 2) podemos observar que a formulação mais preferida pelos julgadores foi a amostra 3 (menor somatório), com a proporção xantana pruni:guar $0,7 \% / 0,7 \%$. Já as formulações menos preferidas foram aquelas com apenas um dos hidrocoloides estudados, guar (amostra 5; maior somatório) ou xantana (amostra 1; segundo maior somatório).

Tabela 2. Somatório das notas do Teste de Ordenação e Índices de Aceitação para a mousse alternativa na variação das concentrações dos hidrocoloides empregados.

\begin{tabular}{|c|c|c|c|c|}
\hline & \multirow{2}{*}{ Amostras } & \multirow{2}{*}{$\begin{array}{l}\text { Somatório da } \\
\text { ordenação* }\end{array}$} & \multicolumn{2}{|c|}{ Índice de aceitabilidade (\%) } \\
\hline & & & Textura & Sabor \\
\hline 1 & $1,4 \%$ xantana & $176^{\mathrm{a}}$ & 68,1 & 72,5 \\
\hline 2 & $1,0 \%$ xantana $+0,4 \%$ guar & $166^{\mathrm{a}}$ & 76,2 & 76,0 \\
\hline 3 & $0,7 \%$ xantana $+0,7 \%$ guar & $146^{\mathrm{a}}$ & 83,6 & 80,4 \\
\hline 4 & $0,4 \%$ xantana $+1,0 \%$ guar & $150^{\mathrm{a}}$ & 78,8 & 78,2 \\
\hline 5 & $1,4 \%$ guar & $186^{\mathrm{a}}$ & 66,9 & 72,5 \\
\hline
\end{tabular}

* valores em ordem decrescente de preferência

$\mathrm{x}_{0}=$ qui-quadrado calculado $=5,96$

$x^{2}=$ qui-quadrado tabelado $(5,55 ; 0,05)=7,78$

letras sobrescritas iguais não diferem estatisticamente $(p<0,05)$

Através do Teste de Aceitação foi possível obter os Índices de Aceitabilidade para cada amostra avaliada; sendo que esses variaram de 66,1 a 83,6\% (gostei ligeiramente a gostei muito) e 72,5 a $80,4 \%$ (gostei regularmente a gostei muito) para a textura e o sabor, respectivamente. Pela análise da Tabela 2 pode-se observar que apenas para o atributo textura foram obtidos Índices de Aceitabilidade menores que $70 \%$ (amostras 1 e 5 consideradas inaceitáveis). Sendo que a amostra que alcançou o maior Índice de Aceitabilidade, para os dois atributos analisados, também foi a mais preferida pelos julgadores no Teste de Ordenação (amostra 3). Dessa forma, a amostra 3, mousse de maracujá alternativa formulada com $0,7 \%$ de xantana pruni e $0,7 \%$ de guar, foi selecionada para os próximos estudos. 
Para fundamentar esses resultados, realizou-se medidas de viscosidade e viscoelasticidade. As curvas de viscosidade apresentaram comportamento pseudoplástico em todas as concentrações utilizadas das gomas estudadas (Figura 1). Como já era esperado, as amostras de mousse contendo maiores concentrações de xantana pruni apresentaram-se mais pseudoplásticas que as amostras com maiores concentrações de goma guar. De acordo com Challen (1994) e Katzbauer (1998), a pseudoplasticidade tende a promover uma diminuição da sensação de gomosidade percebida na boca, colaborando para acentuar outros atributos sensoriais, como o sabor. Segundo esses autores, a xantana exibe baixa viscosidade em taxas de cisalhamento entre 50 e $200 \mathrm{~s}^{-1}$ (taxa da mastigação), o que permite que o sabor do produto seja mais bem percebido. Entretanto, em muitos casos, deve-se utilizar uma combinação de hidrocoloides em determinadas concentrações, a fim de obter a textura desejável; como foi verificado no presente trabalho.

Kuck (2012), ao avaliar a viscosidade do mousse de mirtilo, também verificou que a maior viscosidade, como esperado, foi encontrada na amostra formulada com a maior concentração de xantana e guar.

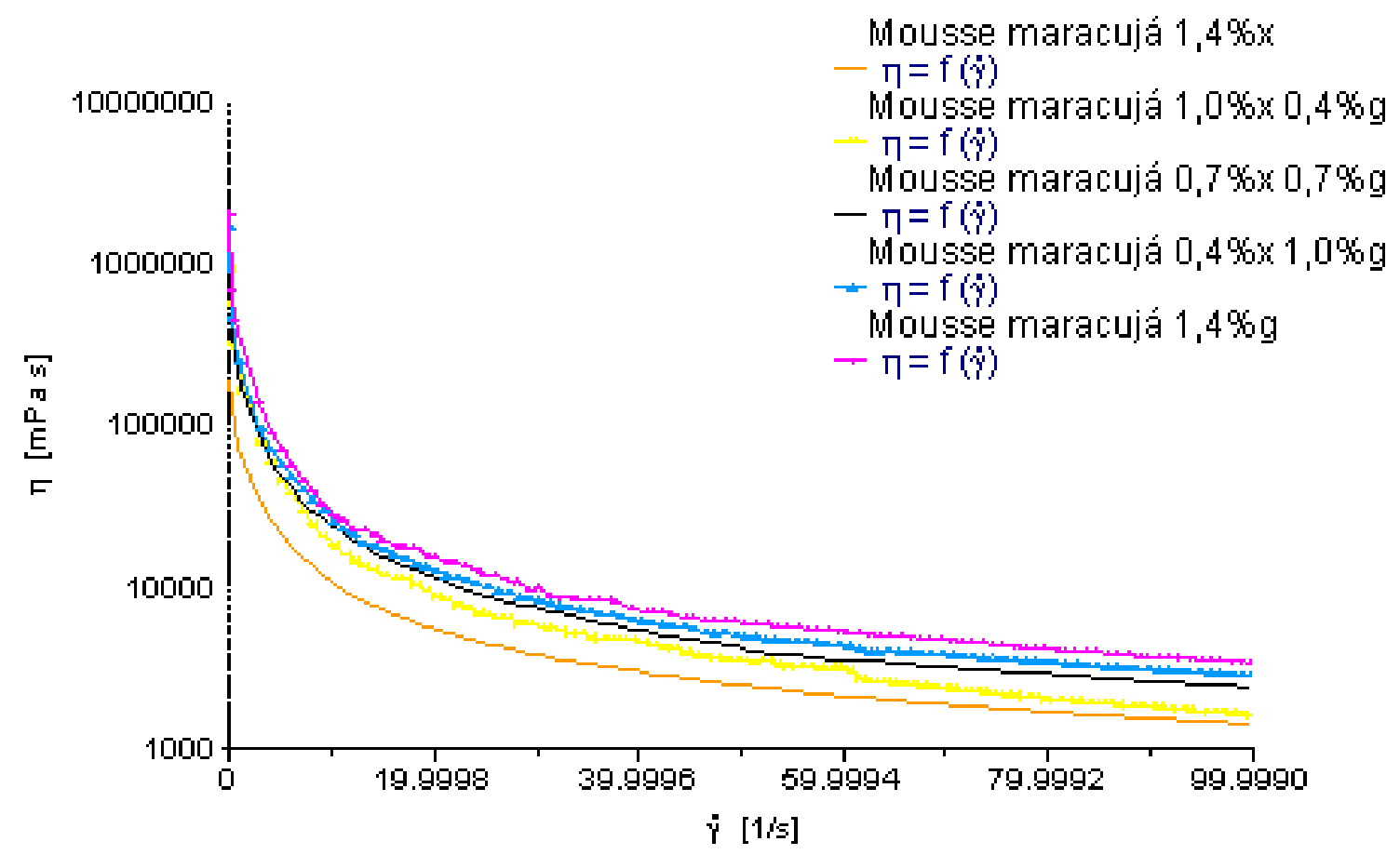

Figura 1. Resultados das medidas rotatórias para as amostras de mousse de maracujá em função da variação das concentrações dos hidrocoloides polissacarídeos ( $\mathrm{x}=$ xantana; $\mathrm{g}$ = guar).

O emprego da guar aumentou tanto o módulo viscoso (G') como o módulo elástico (G") das mousses de maracujá (Figura 2). Quanto maior a concentração de guar utilizada, maior a força ou resistência do gel formado; sendo que a formulação 5 , apenas com guar, foi a menos apreciada, provavelmente devido à excessiva rigidez do gel formado. Por outro lado, a formulação 1, apenas com xantana, originou um gel excessivamente fraco (baixo valor de G"), estrutura não característica de mousse, não sendo, por conseguinte, muito apreciada pelos julgadores; fazendo com que essa amostra obtivesse a segunda menor preferência e Índice de Aceitabilidade. 
Dessa forma, esses resultados indicam que os julgadores apreciam um produto que apresente um equilíbrio nas propriedades de viscosidade e elasticidade, de forma a proporcionar a textura de gel com a cremosidade desejada e apreciada por muitos consumidores. A cremosidade, em termos sensoriais, está relacionada às sensações de textura sentidas e produzidas na boca, sendo, assim, um atributo crítico de qualidade para a aceitação de muitos produtos emulsionados (RICHARDSONHARMAN et al., 2000; TÁRREGA; COSTELL, 2007).

O mesmo comportamento foi observado por Kuck (2012), em que pode-se perceber que o módulo elástico ( $G$ ') de todas as formulações estudadas foi superior ao módulo viscoso (G"), o que representa uma resposta predominantemente elástica, caracterizando comportamento de gel verdadeiro.

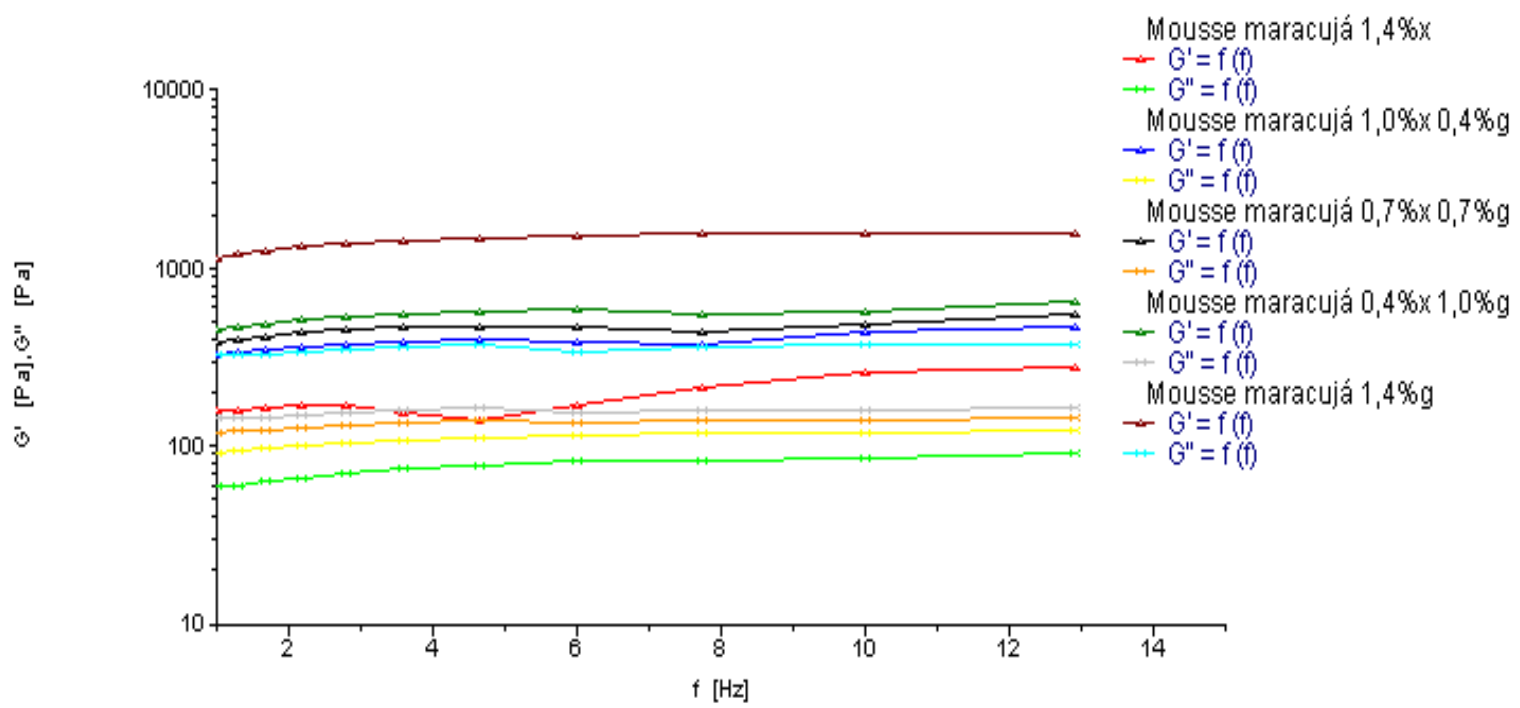

Figura 2. Resultados das medidas oscilatórias para as amostras de mousse de maracujá em função da variação das concentrações dos hidrocoloides polissacarídicos (G' = módulo viscoso; G" = módulo elástico; $x$ = xantana; $g$ = guar).

Após seleção da melhor associação de hidrocoloides na mousse alternativa, $0,7 \%$ de xantana pruni e $0,7 \%$ de guar, estudou-se a melhor combinação das concentrações de açúcar e de suco de maracujá. Na Tabela 3 estão apresentados os resultados para os Testes de Ordenação e de Aceitação para as amostras.

De acordo com a análise estatística, pode-se verificar que as amostras não apresentaram diferença significativa entre elas $\left(x_{0}<x^{2}\right)$, demonstrado igual preferência (Tabela 3). Mesmo assim, observou-se, pelo somatório da ordenação, que os julgadores preferiram a formulação com menor concentração de açúcar e de suco (menor somatório; formulação 2); a formulação menos preferida foi a mousse elaborada com maior concentração de açúcar e suco (maior somatório; formulação 3). A preferência pela formulação 2 deve-se, provavelmente, ao fato de que o suco de maracujá, além de ser responsável pelo aroma, cor e sabor, também está relacionado com a elevação da acidez do produto, característica esta que não foi apreciada pelos julgadores que participaram desta análise sensorial. 
Tabela 3. Somatório das notas do Teste de Ordenação e Índice de Aceitabilidade das mousses alternativas na variação das concentrações de açúcar e de suco de maracujá.

\begin{tabular}{lcccc}
\hline & \multirow{2}{*}{ Amostras } & $\begin{array}{c}\text { Somatório da } \\
\text { ordenação* }\end{array}$ & \multicolumn{2}{c}{ Índice de aceitabilidade (\%) } \\
\cline { 4 - 5 } & & $140^{\mathrm{a}}$ & Textura & Sabor \\
\hline $\mathbf{1}$ & $27 \%$ açúcar + 25\% suco & $127^{\mathrm{a}}$ & 87,1 & 66,5 \\
$\mathbf{2}$ & $27 \%$ açúcar + 22\% suco & $147^{\mathrm{a}}$ & 64,6 & 81,2 \\
$\mathbf{3}$ & $33 \%$ açúcar + 25\% suco & $136^{\mathrm{a}}$ & 77,0 & 63,4 \\
$\mathbf{4}$ & $33 \%$ açúcar + 22\% suco & & 76,2 \\
\hline
\end{tabular}

* valores em ordem decrescente de preferência

$x_{0}=$ qui-quadrado calculado $=2,28$

$x^{2}=$ qui-quadrado tabelado $(4,55 ; 0,05)=6,25$

letras sobrescritas iguais não diferem estatisticamente $(p<0,05)$

Os resultados do Teste de Aceitação corroboraram com esses resultados, visto que, tanto para a textura quanto para o atributo sabor, os menores Índices de Aceitabilidade foram para as amostras com maiores concentração de suco (amostras $1=67,1$ e $66,5 \%$ e amostra $3=64,6$ e $63,4 \%$ ). Além disso, o maior índice de aceitabilidade foi para a amostra mais preferida pelos julgadores (amostra $2=82,4$ e 81,2\%, situandose no ponto da escala em gostei muito).

Após a realização dos 2 ensaios sensoriais, em que foram selecionadas as concentrações dos hidrocoloides, de açúcar e de suco de maracujá para as formulações alternativas, foram confrontadas as formulações alternativas ( $A=$ com xantana pruni e $\mathrm{B}=\mathrm{com}$ xantana comercial) com a formulação tradicional (à base de produtos de origem animal) em um terceiro ensaio sensorial.

A Tabela 4 mostra os somatórios das notas do Teste de Ordenação para as 3 amostras de mousse. Observou-se, através da análise estatística, que houve diferença significativa entre as amostras $\left(x_{0}>x^{2}\right)$, ou seja, houve diferença na preferência dos julgadores. Pelo somatório das notas do Teste de Ordenação, foi observado que a mousse tradicional recebeu a menor pontuação, significando que foi a formulação mais preferida pelos julgadores; entretanto, pelo cálculo do dms (diferença mínima significativa) ao nível de 5\%, a formulação tradicional não diferiu significativamente da formulação alternativa com xantana pruni; podendo-se dizer que, estatisticamente, essas duas formulações foram igualmente preferidas pelos julgadores.

Os Índices de Aceitabilidade (Tabela 5) variam de 56,8 a 92,7\% (entre indiferente e gostei muitíssimo) para a textura e 61,8 a 95,2\% (entre gostei ligeiramente e gostei muitíssimo) para o sabor; sendo que apenas a amostra 3 (formulação alternativa com xantana comercial) foi considerada inaceitável (Índice de Aceitabilidade < 70,0\%). Segundo Cardello et al. (2000), o estudo do comportamento do consumidor tem se baseado em medidas afetivas, como a aceitação e a preferência por determinado produto; entretanto, para Guerrero et al. (2000), também é importante avaliar se o consumidor compraria o produto. Dessa forma, também foi analisada a Intenção de Compra das amostras estudadas, cujas porcentagens variam de 53,4 a 93,8\%, conforme Tabela 5. 
Tabela 4. Somatório das notas do Teste de Ordenação das diferentes formulações de mousse de maracujá.

\begin{tabular}{|c|c|c|c|}
\hline \multirow[b]{2}{*}{ Amostras } & \multicolumn{3}{|c|}{ Somatório da ordenação* } \\
\hline & $\begin{array}{c}\text { Mousse } \\
\text { tradicional (1) }\end{array}$ & $\begin{array}{c}\text { Mousse alternativa } \\
\text { A (2) }\end{array}$ & $\begin{array}{c}\text { Mousse } \\
\text { alternativa B (3) }\end{array}$ \\
\hline Somatório & $98^{\mathrm{C}}$ & $104^{b}$ & $137^{\mathrm{a}}$ \\
\hline Diferença (1) & -- & $6^{\text {ns }}$ & $39^{* *}$ \\
\hline Diferença (2) & $6^{\text {ns }}$ & -- & $33^{* *}$ \\
\hline Diferença (3) & $39^{* *}$ & $33^{* *}$ & -- \\
\hline $\begin{array}{l}\text { valores em ordem } \\
{ }^{*} \text { significativo com } \\
0=\text { qui-quadrado c } \\
2=\text { qui-quadrado ta } \\
\text { ms }=24,28 \\
{ }^{n} \text { não significativo c } \\
b, c \text { letras distintas } \\
=\text { xantana pruni: } \\
=\text { xantana comerc }\end{array}$ & $\begin{array}{l}\text { rescente de prefer } \\
05 \\
\text { ado }=52,53 \\
\text { do }(3,55 ; 0,05)=4,60 \\
p<0,05 \\
\text { rem estatisticamen } \\
\text { yuar }\end{array}$ & $\begin{array}{r} \\
<0,05)\end{array}$ & \\
\hline
\end{tabular}

Tabela 5. Resultados dos Índices de Aceitabilidade e porcentagem de Intenção de Compra das amostras de mousse de maracujá.

\begin{tabular}{llccc}
\hline & Amostras & \multicolumn{2}{c}{ Índice de Aceitabilidade (\%) } & \multirow{2}{*}{$\begin{array}{c}\text { Intenção de } \\
\text { Compra }\end{array}$} \\
\cline { 3 - 4 } & Textura & Sabor & \\
\hline $\mathbf{1}$ & Mousse tradicional & 92,7 & 95,2 & 93,8 \\
$\mathbf{2}$ & Mousse alternativa A* & 86,9 & 82,8 & 85,5 \\
3 & Mousse alternativa B** & 56,8 & 61,8 & 53,4 \\
\hline
\end{tabular}

${ }^{\star} A=$ com xantana pruni:guar

** $\mathrm{B}=$ com xantana comercial:guar

A partir do Teste de Intenção de Compra observou-se que apenas a amostra de mousse de maracujá alternativa formulada com xantana comercial apresentou baixa porcentagem $(53,4 \%)$; sendo consideradas com alta porcentagem as mousses de formulações tradicional $(93,8 \%)$ e alternativa com xantana pruni $(85,5 \%)$. A mousse de maracujá alternativa, formulada com xantana pruni, apresentou Índice de Aceitabilidade e Intenção de Compra semelhantes à mousse tradicional; porém, superiores à mousse com xantana comercial, e com potencial de comercialização.

Segundo Luckow e Delahunty (2004), a maioria dos julgadores avalia a aceitação do produto essencialmente em termos de sabor, ao invés de analisar as características gerais do produto. Em termos de produtos que apresentem soja como um dos ingredientes, essa análise geralmente pode ser prejudicada, devido ao sabor adstringente e aroma pouco agradável que esse ingrediente pode conferir ao produto. 
Entretanto, em nosso trabalho, os resultados evidenciaram um potencial sensorial da formulação proposta que é isenta de ingredientes de origem animal (amostra 2).

Ao avaliarem a intenção de compra de mousse de uva empregando substitutos dos ingredientes de origem animal, Antunes et al. (2014) utilizaram como hidrocoloides xantana e carboximetilcelulose (CMC), tendo a combinação de $0,7 \%$ para ambos a melhor aceitação, recebendo nota 4 que se refere ao "provavelmente compraria"; o ensaio totalizou 30 julgadores. Mesmo com um dos hidrocoloides diferentes, pode-se notar que, em ambos trabalhos, as combinações de concentrações iguais foram bem aceitas pelos julgadores.

No estudo da determinação instrumental da cor, de acordo com Silva, Petter e Schneider (2007), valores positivos e negativos de $a^{*}$ indicam cores vermelhas e verdes, respectivamente. Do mesmo modo, as cores amarelo e azul são demonstrados pela coordenada $b^{*}$ positiva e negativa, respectivamente. L* é uma medida da escala cinza, entre o preto e o branco. O encontro dos três valores gera o ângulo $\mathrm{H}^{\circ}$ que definirá a cor. Dessa forma, os valores da luminosidade $\left(L^{*}\right)$ e das duas coordenadas de cromaticidade $\left(a^{\star}\right.$ e $\left.b^{\star}\right)$ (Tabela 6$)$ indicaram que as três formulações de mousse de maracujá apresentaram coloração amarela, como era esperado.

Tabela 6. Valores de luminosidade ( $L$ ), coordenadas de cromaticidade $a^{\star} e b^{\star}$, valores dos ângulos de cor Hue $\left(\mathrm{H}^{\circ}\right)$ e do parâmetro Croma $\left(\mathrm{C}^{*}\right)$ das mousses de maracujá.

\begin{tabular}{cccccc}
\hline $\begin{array}{c}\text { Formulações } \\
\text { mousses }\end{array}$ & $\mathbf{L}$ & $\mathbf{a}^{*}$ & $\mathbf{b}^{*}$ & $\mathbf{H}^{\mathbf{0}}$ & $\mathbf{C}^{*}$ \\
\hline Tradicional & $81,1^{\mathrm{a}} \pm 0,4$ & $-6,5^{\mathrm{a}} \pm 0,2$ & $33,6^{\mathrm{a}} \pm 0,5$ & $100,9^{\mathrm{a}} \pm 0,4$ & $34,3^{\mathrm{a}} \pm 0,4$ \\
Alternativa $\mathrm{A}^{*}$ & $78,2^{\mathrm{b}} \pm 0,8$ & $-4,5^{\mathrm{b}} \pm 0,1$ & $27,8^{\mathrm{b}} \pm 0,6$ & $99,1^{\mathrm{b}} \pm 0,3$ & $28,1^{\mathrm{b}} \pm 0,6$ \\
Alternativa $\mathrm{B}^{\star \star}$ & $77,7^{\mathrm{c}} \pm 0,2$ & $-4,4^{\mathrm{b}} \pm 0,1$ & $27,8^{\mathrm{b}} \pm 0,3$ & $99,0^{\mathrm{b}} \pm 0,1$ & $28,1^{\mathrm{b}} \pm 0,3$ \\
\hline
\end{tabular}

* $A=$ com xantana pruni

${ }^{* *} \mathrm{~B}=$ com xantana comercial

a, b, c letras distintas na coluna diferem estatisticamente $(p<0,05)$

Pela análise estatística, observou-se que, embora visualmente as amostras de mousse de maracujá apresentassem a mesma coloração, através da análise instrumental associada à análise estatística dos resultados verificou-se diferença significativa no parâmetro $L$ nas três formulações de mousses estudadas (Tabela 6). Nos demais parâmetros $\left(a^{\star}, b^{*}, H^{\circ}\right.$ e $\left.C^{\star}\right)$ verificou-se diferença significativa apenas para a mousse de formulação tradicional; enquanto que não houve diferença significativa entre as duas formulações alternativas.

As amostras apresentaram valores elevados para o parâmetro $L$, que representa a luminosidade; quanto maior for o valor do $L$ (medida da escala cinza, entre o preto e o branco) mais próximo do branco localiza-se, ou seja, mais clara é a amostra. Esse parâmetro foi mais elevado na formulação tradicional, provavelmente devido à maior concentração de proteínas, em função da concentração dos ingredientes empregados nessa formulação, o que acarretou uma maior opacidade ao produto.

As coordenadas de cromaticidade $a^{\star}$ e $b^{\star}$ e o parâmetro $\mathrm{H}^{\circ}$, que é responsável pela tonalidade amarela das amostras, foram proporcionais à quantidade de suco 
empregada às mousses de maracujá. Em função das coordenadas de cromaticidade também foi calculado o parâmetro Croma $\left(C^{*}\right)$, que indicou maior intensidade de cor para a mousse tradicional. No presente trabalho os valores negativos e positivos obtidos para os parâmetros $a^{*}$ e b* indicaram cores verde e amarela, respectivamente; em função desses dois parâmetros calculou-se o ângulo Hue, que correspondeu à tonalidade amarela; e juntamente com o parâmetro L, concluiu-se que as 3 amostras de mousse apresentaram coloração amarelo clara.

Embora tenham sido adicionadas as mesmas concentrações de suco em todas as formulações, houve diferença significativa entre as formulações tradicional e alternativas nesses parâmetros. Tal diferença pode ser atribuída pela presença das gomas, xantana e guar, que podem ter encapsulado algum composto que gera a cor, mascarando-o, de forma a reduzir a tonalidade do produto nas formulações alternativas quando comparado com a tradicional. De acordo com Lucca e Tepper (1994), além de a xantana possuir as características de ser espessante e estabilizante, também apresenta poder encapsulador de compostos.

Kuck (2012) estudou o desenvolvimento de polpa de mirtilo (Vacciunum ashei Reade) e preservação das suas antocianinas para aplicação em alimentos; com a polpa, desenvolveu uma mousse sem a adição de produtos de origem animal, e verificou que o parâmetro $L^{*}$ foi o único que reduziu devido à menor concentração de xantana $e$ guar $(0,4 \% / 0,4 \%)$.

Magalhães e Silva (2018) desenvolveram uma mousse de tamarindo vegano a partir de base de mandioca e de extrato de amêndoas, e verificaram que o parâmetro $L$ reduziu de 86,29 $\pm 0,67$, para a mousse sem adição de tamarindo, para 42,95 $\pm 1,01$, com $100 \%$ da adição do fruto, o que foi esperado pelos autores; para a saturação de cor (C) os valores obtidos foram de $22,75 \pm 0,50$ para a amostra padrão, e de $11,55 \pm$ 0,17 para $100 \%$ da adição, inferindo que o parâmetro teve influência da base do produto. Já para a análise de tonalidade de cor, houve uma redução dos valores de $85,09 \pm 0,09$, da amostra sem adição do tamarindo, para 54,93 $\pm 0,32$, com a adição de $100 \%$, respectivamente. A mesma tendência observou-se no presente trabalho, com a amostra tradicional obtendo valores maiores que as demais estudadas.

\section{Conclusão}

A mousse de maracujá alternativa, formulada com a xantana pruni, apresentou Índice de Aceitabilidade e Intenção de Compra semelhantes à mousse tradicional; porém superiores à mousse com xantana comercial. A mousse alternativa com xantana pruni possui, portanto, potencial para comercialização como sobremesa destinada aos consumidores que não podem ou, por ideologia, não incluem na sua dieta produtos de origem animal.

\section{Referências}

ANTUNES, B. da F. et al. Avaliação da intenção de compra de mousse de uva empregando substitutos dos ingredientes de origem animal. 13 ${ }^{\mathrm{a}}$ Mostra da Produção Universitária, Universidade Federal de Rio Grande, Rio Grande, 2014.

CARDELLO, A. V. et al. Predictors of food acceptance, consumption and satisfaction in specific eating situations. Food Quality and Preference, v. 11, p. 201-216, 2000. https://doi.org/10.1016/S0950-3293(99)00055-5. 
CHALLEN, I. A. Xanthan gum: a multifunctional stabilizer for food products. In: NISHINARI, K.; DOI, E. (Editores). Food hydrocolloids: structure, properties, and functions. New York: Plenum Press, p. 135-140, 1994. https://doi.org/10.1007/978-14615-2486-1.

DEMIRCI, Z. O.; YILMAZ, I.; DEMIRCI, A. S. Effects of xanthan, guar, carrageenan and locust bean gum addition on physical, chemical and sensory properties of meatballs. Journal of Food Science and Technology, v. 51, n. 5, p. 936-942, 2014. https://doi.org/10.1007/s13197-011-0588-5.

DI COSTANZO, M.; CANANI,R. B. Lactose intolerance: common misunderstandings. Annals of nutrition \& metabolism, v. 73, p. 30-37, 2018. https://doi.org/10.1159/000493669.

DICKINSON, E. Hydrocolloids at interfaces and the influence on the properties of dispersed systems. Food Hydrocolloids, v. 17, p. 25-39, 2003. https://doi.org/10.1016/S0268-005X(01)00120-5.

FOLEGATTI, M. L. S. Estudo do efeito do uso de diferentes agentes aerantes e gelificantes e do processamento nas características físicas e sensoriais e na estabilidade do produto mousse de maracujá. 2001. 203f. Tese (Doutorado em Ciência de Alimentos), Programa de Pós-Graduação em Ciência de Alimentos, Universidade Estadual de Campinas, Campinas, 2001.

GARCÍA-OCHOA, F. et al. Xantana gum: production, recovery, and properties. Biotechnology Advances, v.18, p. 549-579, 2000. https://doi.org/10.1016/S07349750(00)00050-1.

GUERRERO, L. et al. Consumer attitude towards store brands. Food Quality and Preference, v. 11, p. 387-395, 2000. https://doi.org/10.1016/S0950-3293(00)00012-4.

IBGE. Produção Agrícola Municipal, 2019. Disponível em: <https://sidra.ibge.gov.br/tabela/5457\#resultado>. Acesso em: 10 dez. 2020.

LUCKOW, T.; DELAHUNTY, C. Which juice is healthier? A consumer study of probiotic non-dairy juice drinks. Food Quality and Preference. v. 15, p. 751-759, 2004. https://doi.org/10.1016/j.foodqual.2003.12.007.

KATZBAUER, B. Properties and applications of xanthan gum. Polymer Degradation and Stability, v. 59, p. 81-84, 1998. https://doi.org/10.1016/S0141-3910(97)00180-8.

KUCK, L. S. Desenvolvimento de polpa de mirtilo (Vaccinium ashei Reade) e preservação das suas antocianinas para aplicação em alimentos. 2012. $126 \mathrm{f}$. Dissertação (Mestrado em Ciência e Tecnologia de Alimentos), Programa de PósGraduação em Ciência e Tecnologia de Alimentos, Universidade Federal de Pelotas, Pelotas, 2012.

LAUFFER, M. L. et al. Estudo da aplicação de diferentes hidrocoloides para elaboração de mousse de maracujá livre de ingredientes de origem animal. XIX Congresso de Iniciação Científica da Universidade Federal de Pelotas, Anais do XIX Congresso de Iniciação Científica da Universidade Federal de Pelotas, Pelotas, 2010.

LILLY, V.G.; WILSON, H. A.; LEACH, J. G. Bacterial polysaccharides II. Laboratory Scale production of polysaccharides by species $X$. campestris. Applied Microbiology, v.6, p.105-109, 1958. https://www.ncbi.nlm.nih.gov/pmc/articles/PMC1057366/. 
LONDERO, D. S. "Você é aquilo que você come": o veganismo enquanto estilo de vida e ativismo político. 2019. 113 f. Dissertação (Mestrado em Ciências Sociais), Programa de Pós-Graduação em Ciências Sociais, Universidade Federal de Santa Maria, Santa Maria, 2019.

LUCCA, P.; TEPPER, B. Fat replacers and the functionality of fat foods. Trends in Food Science \& Technology, v. 5, p. 12-19, 1994. https://doi.org/10.1016/09242244(94)90043-4.

LUVIELMO, M.; SCAMPARINI, A. R. P. Goma xantana: produção, recuperação, propriedades e aplicação. Estudos Tecnológicos, v. 5, n. 1, p. 50-67, 2009. https://doi.org/10.4013/ete.2009.51.04.

MAGALHÃES, D. A.; da SILVA, F. M. Desenvolvimento de mousse de tamarindo vegano a partir de base de mandioca e de extrato de amêndoas: Caracterização microbiológica, físico-química e como carreador de probiótico. 2018. 46 f. Trabalho de Conclusão de Curso (Graduação em Nutrição), Faculdade de Ciências da Saúde, Universidade Federal de Brasília, Brasília, 2018.

MCCLEMENTS, D. J. Food emulsions; principles, practice, and techniques. Boca Raton, FL: CRC Press, 2005.

OHATA, S. M. et al. Adição de concentrado protéico de soro (CPS) em mousse de maracujá. Revista Ciências Exatas e Naturais, v. 7, n. 1, p. 53-66, 2005.

PARASKEVOPOULOU, A. et al. Influence of polysaccharide addition on stability of a cheese whey kefir-milk mixture. Food Hydrocolloids, v. 17, p. 615-620, 2003. https://doi.org/10.1016/S0268-005X(02)00122-4.

RICHARDSON-HARMAN, N. J. et al. Mapping consumer perceptions of creaminess and liking for liquid dairy products. Food Quality Preference, v. 11, p. 239-246, 2000. https://doi.org/10.1016/S0950-3293(99)00060-9.

ROJAS, J. A.; ROSELL C. M.; BARBER, C. B. Pasting properties of different wheat flour-hydrocolloid systems. Food Hydrocolloids, v. 13, p. 27-33, 1998.

SILVA, R. A.; PETTER, C. O.; SCHNEIDER, I. A.H. Avaliação da perda da coloração artificial de ágatas. REM: Revista Escola de Minas, v.60, p.477-482, 2007. https://doi.org/10.1590/S0370-44672007000300007.

SILVA, R. R. et al. Efeito da utilização de gomas na viscosidade e nas características sensoriais de shake à base de farinha de banana verde. Brazilian Journal of Food Technology, v. 21, e2016052, 2018. https://doi.org/10.1590/1981-6723.5216.

TÁRREGA, A.; COSTELL, E. Color and consistency of semi-solid dairy desserts: Instrumental and sensory measurements. Journal of Food Engineering, v. 78, p.655661, 2007. https://doi.org/10.1016/j.jfoodeng.2005.11.003.

VENDRUSCOLO, C. T.; MOREIRA, A. S.; VENDRUSCOLO, J. L. S. Processo de produção de biopolímero tipo xantana, biopolímero obtido, seus usos; meio de cultura 
para crescimento de xanthomonas e uso da mesma para produção de biopolímero. World Intellectual Property Organization. 2006.

ZHANG, Y. et al. Stability and color characteristics of PEF treated cyaniding-3-glicoside during storage. Food Chemistry, v. 106, p. 669-679, 2008. https://doi.org/10.1016/j.foodchem.2007.06.030.

\section{Agradecimentos}

Agradecemos às agências de fomento CAPES, FAPERGS e CNPq.

\section{Autores}

Fernanda Germano Alves Gautério ${ }^{1}$, Mariane Igansi Alves ${ }^{2}$, Matheus Ludtke Lauffer ${ }^{3}$, Karine Laste Macagnan ${ }^{4}$, Claire Tondo Vendruscolo ${ }^{5}$, Angelita da Silveira Moreira ${ }^{3, *}$

1. Curso de Pós-graduação Lato Sensu em Ciência dos Alimentos, Centro de Ciências Químicas, Farmacêuticas e de Alimentos, Universidade Federal de Pelotas, Campus Universitário, Caixa Postal, 354, 96010-900, Pelotas, RS, Brasil; Curso de Engenharia de Alimentos, Universidade Federal do Pampa, Campus Bagé, Av. Maria Anunciação Gomes de Godoy, 1650, Bairro Malafaia, 96413-172, Bagé, RS, Brasil.

2. Departamento de Ciência e Tecnologia Agroindustrial, Universidade Federal de Pelotas, Universidade Federal de Pelotas, Campus Universitário, Caixa Postal, 354, 96010-900, Pelotas, RS, Brasil.

3. Centro de Ciências Químicas, Farmacêuticas e de Alimentos, Universidade Federal de Pelotas, Campus Universitário, Caixa Postal, 354, 96010-900, Pelotas, RS, Brasil.

4. Centro de Desenvolvimento Tecnológico, Universidade Federal de Pelotas, Campus Universitário, Caixa Postal, 354, 96010-900, Pelotas, RS, Brasil.

5. Biopolix Materiais Tecnológicos Ltda., Supera Parque Tecnológico, Av. Dra. Nadir Águiar, 1805, Jardim Dr. Paulo Gomes Romeo, 14056-680, Ribeirão Preto, SP, Brasil.

* Autor para correspondência: angelitadasilveiramoreira@gmail.com 